\title{
Computation of Fingertip Positions for a Form-Closure Grasp*
}

\author{
Dan Ding Yun-Hui Liu \\ Dept. of Auto. and Computer-Aided Eng. \\ The Chinese Univ. of Hong Kong \\ Shatin, N. T., Hong Kong
}

\author{
Jianwei Zhang Alois Knoll \\ Faculty of Technology \\ Univ. of Bielefeld \\ Bielefeld, Germany
}

\begin{abstract}
This paper proposes a simple and efficient algorithm for computing a form-closure grasp on a $3 \mathrm{D}$ polyhedral object. This algorithm searches for a form-closure grasp from a "good" initial grasp in a promising search direction that pulls the convex hull of the primitive contact wrenches towards the origin of the wrench space. The "good" initial grasp is a set of contact points that minimizes the distance between the origin and the centroid of the primitive contact wrenches, and can be calculated by the quadratic programming. The local promising search direction at every step is readily determined by the ray-shooting based qualitative test algorithm developed in our early work. By using the "good" initial grasp, the iteration times of search can be significantly reduced so that a form-closure grasp can be found more efficiently. Since the algorithm adopts a local search strategy, its computational cost is less dependent on the complexity of the object surface. Finally, the algorithm has been implemented and its efficiency has been ascertained by three examples.
\end{abstract}

\section{Introduction}

In the context of manipulation, it might be desirable to have a multi-fingered robot hand rather than carrying a specialized gripper for each operation to be performed. For a multifingered grasp, an important property is the stability of the resulting grasp, which is characterized by form-closure. A grasp is considered to be of form-closure type if it is possible to apply forces and torques at the contact points so that any external forces and torques can be balanced [1][5]. Salisbury and Roth[5] have demonstrated that a necessary and sufficient condition for form-closure is that the primitive contact wrenches resulted by contact forces positively span the entire wrench space. This condition is equivalent to that the origin of the wrench space lies strictly inside the convex hull of the primitive contact wrenches[4].

*This work is supported in part by the Hong Kong Research Grant Council under grants CUHK4151/97E and the German/HK joint research scheme. Email: yhliu@acae.cuhk.edu.hk
The synthesis of a form-closure grasp involves two steps. First, the qualitative test for the form-closure property. Many works have been done [3] [7]. Liu[3] demonstrated that querying whether the origin of the wrench space lies inside the convex hull of the primitive contact wrenches is equivalent to a rayshooting problem, which is dual to a LP problem. The developed algorithm is applicable to $2 \mathrm{D}$ and $3 \mathrm{D}$ grasps with any number of contacts.

The second step is to calculate the contact points that ensure the form-closure property. Despite the huge effort, up to now, there is no efficient and complete algorithms to tackle the problem of calculating a form-closure grasp, especially in 3D cases due to the high dimension of the grasp space and the nonlinearity of the commonly accepted contact friction models. Ponce and Sudsang showed that fourfinger force-closure grasps fall into three categories: concurrent, pencil and regulus grasps and proposed techniques to compute them [6]. Recently, the authors developed an algorithm to compute contact points on the object surfaces yielding a form-closure grasp [8]. The algorithm searchs for a set of feasible grasp points from a set of randomly selected contact points.

In this paper, an efficient and simple algorithm is proposed for computation of 3D form-closure grasps. For a non-form-closure grasp, a local search direction is calculated in each iteration along which the convex hull of the primitive contact wrenches is moved towards the origin until the origin is completely contained by the hull. The motion is formulated as a set of quadratic programming problems. To reduce the iteration numbers, a "good" set of initial grip points is required, which can be calculated by another quadratic programming with the objective function of minimizing the distance between the centroid of the primitive contact wrenches and the origin. Finally, the performance of the algorithm is confirmed with simulations. It should be noted that the proposed approach is applicable to any number of contacts and it is not necessary to provide a random initial grasp. 


\section{Form-Closure Grasps}

Assume that $n$ hard fingers are to grasp a polyhedral object with friction coefficient $\mu$ at the grip points. To ensure non-slipping at the contact point of finger $i$, the grasping force $f_{i}$ must be within the friction cone (Fig.2), which can be linearized by a polyhedron convex cone with $m$ sides for simplification. Under this approximation, the grasping force $f_{i}$ can

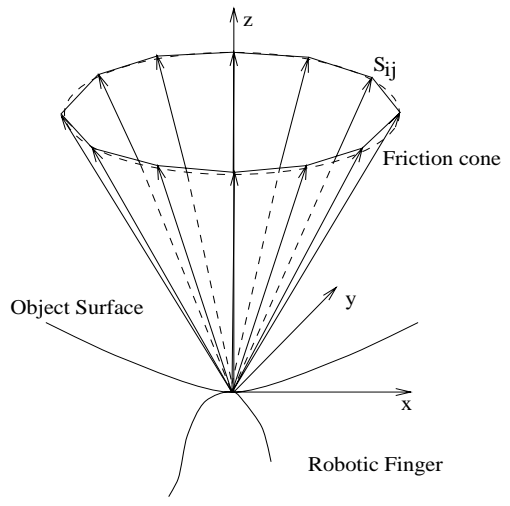

Figure 1: The friction cone at a grasping point.

be represented as

$$
f_{i}=\sum_{j=1}^{m} \alpha_{i j} \vec{s}_{i j}, \quad \alpha_{i j} \geq 0
$$

where $\vec{s}_{i j}$ represents the $j$-th edge vector of the polyhedral convex cone. Coefficients $\alpha_{i j}$ are nonnegative constants. The force and torque, corresponding to the grasping force $f_{i}$, applied at the center of mass of the object is given by

$$
\underline{w}_{i}=\left(\begin{array}{c}
f_{i} \\
\tau_{i}
\end{array}\right)=\left(\begin{array}{c}
f_{i} \\
r_{i} \times f_{i}
\end{array}\right)
$$

where $r_{i}$ denotes the position vector of the $i$-th grasping point w.r.t. the object coordinate frame at the center of mass. The combination $\underline{w}_{i}$ of the force $f_{i}$ and moment $\tau_{i}$ is called wrench. Substituting eq. (1) into eq. (2) derives

$$
\underline{w}_{i}=\sum_{j=1}^{m} \alpha_{i j} w_{i j}
$$

where

$$
w_{i j}=\left(\begin{array}{c}
\vec{s}_{i j} \\
r_{i} \times \vec{s}_{i j}
\end{array}\right)
$$

$w_{i j}$ is called primitive contact wrenches of the finger. The net wrench applied at the object by the fingers is

$$
\underline{w}_{n e t}=\sum_{i=1}^{n} \sum_{j=1}^{m} \alpha_{i j} w_{i j}=W \alpha
$$

where $W$ and $\alpha$ are given by

$$
\begin{aligned}
& W=\left(w_{11}, w_{12}, \ldots, w_{1 m}, \ldots, w_{n 1}, w_{n 2}, \ldots, w_{n m}\right) \\
& \alpha=\left(\alpha_{11}, \alpha_{12}, \ldots, \alpha_{1 m}, \ldots, \alpha_{n 1}, \alpha_{n 2}, \ldots, \alpha_{n m}\right)^{T}
\end{aligned}
$$

$W$ is a $6 \times N$ matrix called wrench matrix where $N=n m$ and its column vectors are the primitive contact wrenches.

Definition 1 Suppose that a $n$-finger frictional grasp is given. For any external wrench $\underline{w}_{\text {ext }}$ applied at the object, if it is always possible to find an $\alpha$ with $\alpha_{i} \geq 0$ so that

$$
W \alpha+\underline{w}_{e x t}=0,
$$

the grasp is said to be form-closure.

It is well-known that a form-closure grasp is equivalent to that the origin of the wrench space $R^{6}$ lies exactly inside the convex hull of the primitive contact wrenches[5].

The problem addressed in this paper is to calculate a form-closure grasp on a given polyhedral object.

\section{Calculation of Form-Closure Grasp}

\subsection{Outline of the algorithm}

The grasp computation algorithm works in the following simple way. The algorithm first calculates a set of points as the initial grasp that minimizes the distance between the origin of the wrench space and the centroid of the primitive contact wrenches by the convex quadratic programming. If the grasp calculated satisfies the form-closure property, the algorithm ends; otherwise, it searches for a form-closure grasp locally in a proper direction. The local search direction is determined based on the qualitative test algorithm developed by Liu [3]. As shown in Fig. 2, the ray from the interior point $P$ of the convex hull of the primitive contact wrenches to the origin $O$ of the wrench space intersects the convex hull at point $Q$. If the distance $\|P O\|$ is strictly smaller than $\|P Q\|$, the grasp is form-closure.

For a non-form-closure grasp, if the primitive contact wrenches move at a step along the normal vector of the facet intersected by the ray $P O$, the convex hull will approach the origin iteration by iteration until the origin is completely contained by the hull. The change of grasp points on the object surfaces corresponding to the desired motion of the primitive contact wrenches can be calculated by a quadratic programming problem. 


\subsection{Qualitative Test and Local Search Direction}

The qualitative test algorithm, proposed in our early work [3], is used to test whether the convex hull of the primitive contact wrenches contains the origin as well as to calculate the local search direction in each iteration. We brief the idea below and the details can be referred to [3].

Theorem 1 Suppose that a n-finger frictional grasp is given. Denote the convex hull of the primitive contact wrenches $w_{i}$ by $H(W)$. Let point $P$ be the centroid of the primitive contact wrenches. The ray from $P$ to the origin $O$ of the wrench space $R^{6}$ intersects $H(W)$ in a point $Q$ only. A form-closure grasp is equivalent to that the distance $d_{1}$ between points $P$ and $Q$ is strictly larger than the distance $d_{2}$ between points $P$ and $O$ (Fig.2).

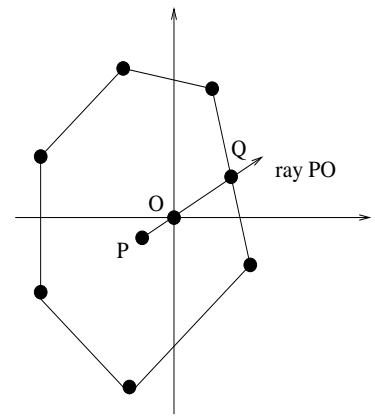

(a)

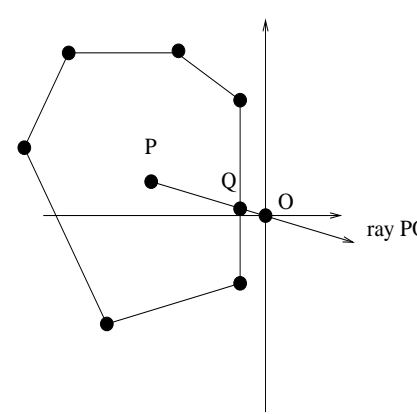

(b)
Figure 2: A form-closure grasp (a) and a non-formclosure grasp (b).

The centroid $P$ is calculated as follows:

$$
P=\frac{1}{N} \sum_{i=1}^{N} w_{i}
$$

Denote the direction vector of the ray $P O$ by $t$. To find the intersection of $H(W)$ with the ray $t$, we need to find the facet of the convex hull intersected by the ray, which is closely related to the rayshooting problem and is equivalent to a problem of maximizing the objective function

$$
z=t^{T} x
$$

subject to the constraints:

$$
\left(w_{i}-P\right)^{T} x \leq 1 \quad i=1,2, \ldots, N
$$

The details can be found in [3]. Suppose that the optimal solution of the linear programming problem is $\vec{e}=\left(e_{1}, e_{2}, \ldots e_{6}\right)$. Then the facet $E$ of $H(W)$ intersected by the ray $t$ is

$$
e_{1} x_{1}+e_{2} x_{2}+e_{3} x_{3}+e_{4} x_{4}+e_{5} x_{5}+e_{6} x_{6}=1 \text {. }
$$

The intersection point $Q$ of $H(W)$ with the ray $P O$ is the intersection of the hyperplane defined by (8) with the ray $P O$.

The normal vector $\vec{e}$ of the facet $E$ is used as the local search direction along which the convex hull of the primitive contact wrenches are moved in each iteration.

\subsection{Heuristic Search for Grip Points Ensuring Form-Closure}

The motion of the primitive contact wrenches along the local search direction $\vec{e}=\left(e_{1}, e_{2}, \ldots, e_{6}\right)$ is realized as follows: for each primitive contact wrench $w_{i}^{k}$ at the $k-t h$ iteration, we select a desired point $w_{i}^{d}$ at the $(k+1)-t h$ iteration, which is obtained by moving $w_{i}^{k}$ along the direction parallel to the normal vector $\vec{e}$ at a step size $\alpha$, i.e.

$$
w_{i}^{d}=w_{i}^{k}+\alpha * \vec{e}
$$

In actual cases, the primitive contact wrenches cannot follow the direction $\vec{e}$ exactly due to the fact that the first three components(force vector) of each primitive contact wrench are constant, and thus deviations from the direction $\vec{e}$ always arise no matter how the wrenches are moved. To solve the problem, we determine the grasp point $r_{j}$ on the surface by the following quadratic programming problem.

$$
\min _{r \in R^{3 n}} \sum_{i=1}^{N}\left(w_{i}^{k+1}(r)-w_{i}^{d}\right)^{2}
$$

subject to

$$
\begin{gathered}
\left(w_{i}^{k+1}(r)-w_{i}^{k}\right) \bullet \vec{e} \geq 0, \quad i=1,2, \ldots, N \\
F_{j}\left(r_{j}\right)=0 \quad j=1,2, \ldots, n \\
N_{o r m} l \bullet r_{j} \geq 0 \quad l=1,2, \ldots t_{j}, \quad j=1,2, \ldots, n
\end{gathered}
$$

where $w_{i}^{k}$ and $w_{i}^{k+1}$ denote the primitive contact wrench of $k-t h$ and $(k+1)-t h$ iteration. The objective function is chosen to minimize the distance between the actual primitive contact wrenches and the desired ones. The first linear constraint eq. (11) denotes that each primitive contact wrench should move along the direction with less than 90 degrees to the ray $\vec{e}$. $r_{j} \in R^{3}$ is the position vector associated with face $j$. The constraints eq. (12) and eq. (13) ensure that the position vectors $r$ calculated should satisfy the equation of corresponding faces and remain inside respectively, where $t_{j}$ is the edge number of the $j-t h$ face and Norm $_{j l}$ denotes the internal norm of the $l-t h$ boundary face of the cone formed by the origin of the object frame(cone vertex) and the edges of face $j$. 




Figure 3: Primitive contact wrenches moving along the normal vector $\vec{e}$.

\subsection{Calculation of A Good Set of Ini- tial Grip Points}

The heuristic search procedure requires a set of initial grip points from which the algorithm starts to search for a feasible solution. If the initial grip points are not 'good' enough or the stepsize in each iteration is not proper, the iteration number may be large before the algorithm terminates with a feasible solution. In this section, we choose a set of points as the initial grasp points that minimize the distance between the centroid of the primitive contact wrenches and the origin of the wrench space.

The centroid $P$ of the primitive contact wrenches can be represented as $A+B r$, where $\mathrm{A}$ and $\mathrm{B}$ are constant matrices with dimension $6 \times 1$ and $6 \times 3 n$ respectively, and $r=\left[r_{1}, \ldots, r_{n}\right]$ denotes $3 n \times 1$ position vector which defines a set of grip points. The computation of the "good" set of initial grasp points can be formulated as the quadratic programming problem as follows:

$$
\min _{r \in R^{3 n}}(A+B r)^{T}(A+B r)
$$

which is also subject to the two constraints eq. (12) and (13).

The quadratic form in the objective function eq. (14) can be written as $r^{T} B^{T} B r$, where $B^{T} B$ is positive semidefinite. And any positive semidefinite quadratic form $q(x) \equiv x^{T} D x$, where $D$ is symmetric, is a convex function over all of $E^{n}$ [10]. Therefore, the objective function is convex. Moreover, the constraints are all linear and thus convex also, so the above quadratic programming problem is a convex programming problem and the solution is a global one. In other words, the set of initial grip points calculated through the QP problem above ensure a global minima of the distance between the centroid and the origin, which increases the robustness of the grasp, so in this sense, the position vector calculated are optimal. Furthermore, as the "good" set of initial grip points already stays as closely as possible to the origin, even if it cannot yield form-closure, it will take less time to find a proper solution.

\subsection{The Algorithm Description}

The discussion above is summarized as the following algorithm:

(1)Calculate the two constant matrices $A$ and $B$ so that the centroid $P$ can be represented.

(2)Solve the quadratic programming problem eq.(14) to obtain a "good" set of initial grip points. Use the qualitative test algorithm to check whether the grasp obtained is formclosure. If so, the program ends with proper solution found.

(3)Calculate the desired primitive contact wrenches $w_{i}^{d}$ and the local search direction $\vec{e}$.

(4)By the quadratic programming eq.(10-13), obtain the new position vectors $r$ and then calculate the new primitive contact points $w_{i}^{k+1}$.

(5)If form-closure cannot be achieved, turn to (3). Otherwise, the algorithm ends with proper solution found.

The main advantage of the algorithm proposed here lies in two aspects. First, the solution obtained by the algorithm is optimal to the extent, as it tries to coincident the origin and the centroid of the primitive contact wrenches, which can decrease the effect of gravitational and inertial forces during the motion of the robot and achieves a robust grasp. Second, due to the "good" set of initial grip points, the iteration number for the heuristic searching procedure is greatly reduced and thus the computation time is greatly shortened, which is demonstrated by the examples in the next section. The main disadvantage is that the algorithm is incomplete due to the local search, i.e. the algorithm has no way to judge whether the problem has solutions and thus when the algorithm fails to find a feasible solution, there is no guarantee that there is none for the problem.

\section{Implementation}

We have implemented the proposed algorithm using Matlab on a Sun SPARC5 workstation and verified its computational efficiency by several examples.

\section{Example 1:}

The example concerns a four-finger grasp of a cube shown in Fig.(4). The friction coefficient $\mu=0.1$ and the friction cone is linearized by 8 segments. 
The "good" initial grip points calculated through eq. (14) can satisfy the form-closure condition (see Fig.4). The final grip points calculated are as follows: $r_{1}=(2.0,0.0,0.0)^{T}, r_{2}=(0.0,2.0,0.0)^{T}, r_{3}=$ $(0.0,0.0,2.0)^{T}, r_{4}=(0.0,-2.0,0.0)^{T}$. However, if we randomly select the initial grip points $r_{i}$ as: $r_{1}=(2.0,1.9,-1.8)^{T}, r_{2}=(-1.9,2.0,-1.8)^{T}, r_{3}=$ $(1.5,1.8,2.0)^{T}, r_{4}=(1.9,-2.0,1.9)^{T}$. A feasible solution can be found after 7 iterations within $3.89 \mathrm{~s}$ when the stepsize is set 0.5 .

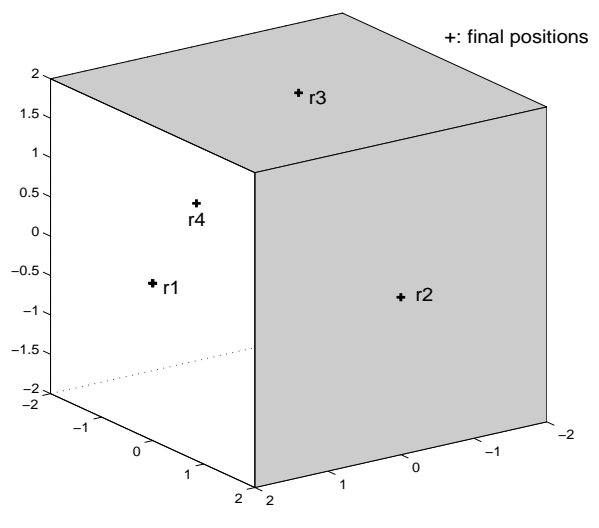

Figure 4: Example 1.

\section{Example 2:}

The second example is about a three-finger grasp shown in Fig.(6). The friction coefficient $\mu=$ 1.3 and the friction cone is linearized by $8 \mathrm{seg}-$ ments. We first obtain the "good" initial grip points $r_{1}=(0.0,0.52,5.0)^{T}, r_{2}=(0.67,4.2,0.16)^{T}, r_{3}=$ $(0.0,-3.9,0.28)^{T}$, from which the algorithm starts to find a feasible solution after 8 iterations within $1.93 \mathrm{~s}$ (Fig. 6). The distance difference $\|P O\|-\|P Q\|$ is plotted vs the iteration number(Fig. 5). However, if we randomly choose the initial grip points as $r_{1}=(-1.5,2.4,5)^{T}, r_{2}=(1.0,4.0,1.0)^{T}, r_{3}=$ $(2.0,-4.0,0.0)^{T}$, by the algorithm with a random initial grasp and the convex hull is moved along the ray $P O$, no feasible solution can be found and the distance $\|P O\|$ remains unchanged after several iterations. When the convex hull is moved along $\vec{e}$, after 15 iterations(Fig. 7), the proper position vector can be found within $6.03 \mathrm{~s}$ when the stepsize is 1.5. The position vector calculated are as follows: $r_{1}=(-0.68,1.05,5.0)^{T}, r_{2}=$ $(-0.2,4.0,2.19)^{T}, r_{3}=(0.81,-3.78,1.15)^{T}$. In addition, if we change the friction coefficient $\mu=1.25$, then there is no feasible solution found. The distance difference $\|P O\|-\|P Q\|$ shows the descending trend at first, but gets rise and then osciliates(Fig. 8 ). The negative value of the distance difference corresponding to a feasible solution can never be achieved.

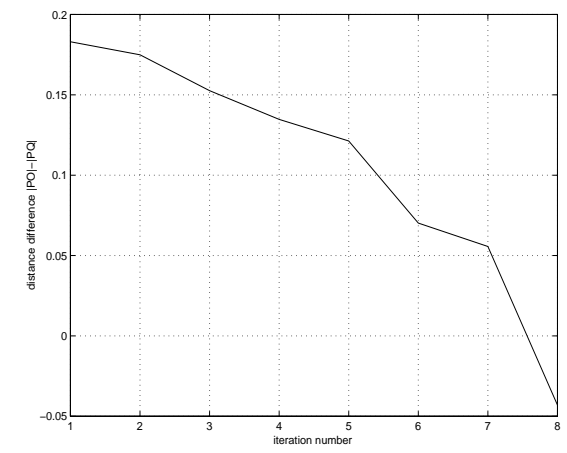

Figure 5: Distance Difference $\|P O\|-\|P Q\|$

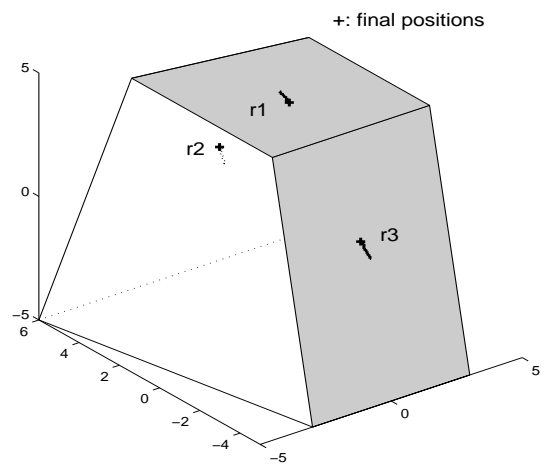

Figure 6: Example 2.

\section{Example 3:}

The third example concerns a four-finger grasp of a tetrahedron shown in Fig.(9). The friction coefficient $\mu=0.2$ and the friction cone is linearized by 8 segments. Using the algorithm with a "good" initial grasp, the final position vectors $r_{1}=(1.26,-0.10,3.00)^{T}, r_{2}=$ $(3.00,-0.05,0.05)^{T}, r_{3}=(1.13,3.00,0.34)^{T}, r_{4}=$ $(1.76,0.84,0.40)^{T}$ can be obtained after 4 iterations within $0.99 \mathrm{~s}$. If the initial position vectors are given as $r_{1}=(1.2,-1.2,3.0)^{T}, r_{2}=(3.0,2.2,-1.2)^{T}, r_{3}=$ $(0.2,3.0,2.6)^{T}, r_{4}=(1.0,1.0,1.0)^{T}$, by the algorithm with a random initial grasp and when the convex hull is moved along $\vec{e}$, proper solutions can be found after 8 iterations and it takes 3.34s when the stepsize is $1.0 \mathrm{~s}$.

Table 1: Comparison of computation time and iteration number of the approach with random initial grasp(Approach 1) and that with "good" initial grasp(Approach 2).

\begin{tabular}{|c|c|c|c|}
\hline Time $($ s)/Iteration & $e x .1$ & $e x .2$ & $e x .3$ \\
\hline Approach 1 & $3.89 / 7$ & $6.03 / 15$ & $3.34 / 8$ \\
\hline Approach 2 & $0.21 / 1$ & $1.93 / 9$ & $0.99 / 5$ \\
\hline
\end{tabular}




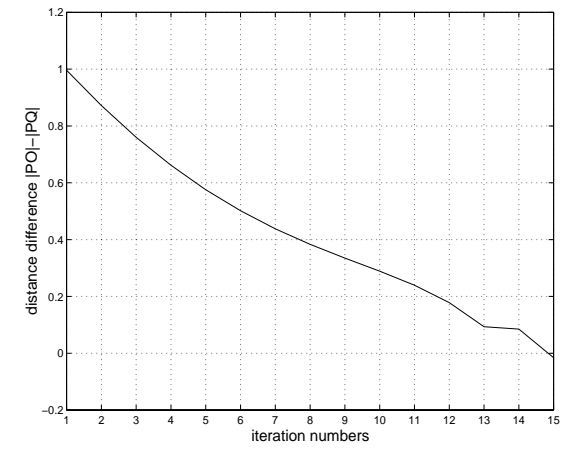

Figure 7: Iteration numbers by using random initial grip points

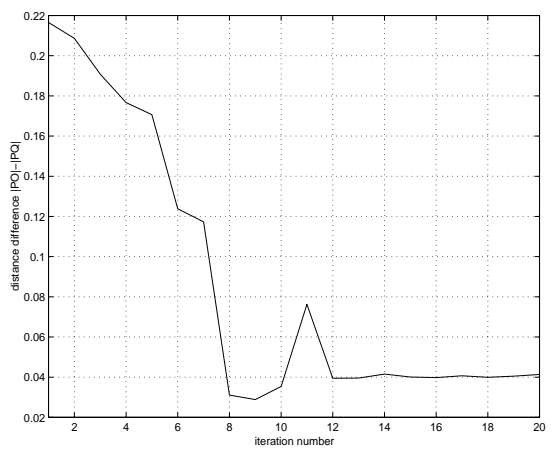

Figure 8: Distance difference when no feasible solutions can be found

It should be noted that the iteration number for Approach 2 consists of the iteration number for the local search and that for getting initial "good" grasp, which always equals one.

\section{Conclusions}

This paper addressed the problem of computing contact points yielding form-closure for a given set of faces. For a non-form-closure grasp, we first formulate a quadratic programming problem to shorten the distance between the centroid of the primitive contact wrenches and the origin of the wrench space. Furthermore, another sequence of quadratic programming problems are formulated to move the primitive contact wrenches at a fixed step along the direction $\vec{e}$ as closely as possible, so that the origin will be contained by the convex hull eventually. We have implemented the algorithm and demonstrated its efficiency by 3 numerical examples. The result can be applied to obtain contact points when planning regrasp sequences.

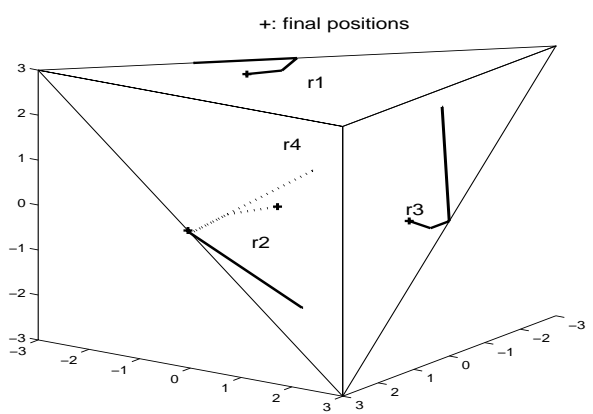

Figure 9: Example 3.

\section{References}

[1] A. Bicchi, "On the closure properties of robotics grasping," Int. Journal of Robotics Research, vol. 14, no. 4, pp. 319-334, 1995.

[2] D. Ding, Y. H. Liu, and S. Wang, "Computing 3-D optimal form-closure grasps", Proc. IEEE Int. Conf. on Robotics and Automation, 2000.

[3] Y. H. Liu, "Qualitative test and force optimization of 3D frictional form-closure grasps using linear programming," IEEE Trans. on Robotics and Automation, vol.15, no. 1, pp. 163-173, 1999.

[4] B. Mishra, J. T. Schwartz, and M. Sharir, "On the existence and synthesis of multifinger positive grips," Algorithmica, Special Issue: Robotics, vol. 2, no. 4, pp. 541-558, 1987.

[5] J. K. Salisbury and B. Roth, "Kinematic and force analysis of articulated hands," $A S M E$ Journal of Mechanism, Transmissions, Automation, Design, vol. 105, pp. 33-41, 1982.

[6] A. Sudsang and J. Ponce, "New Technique for computing four-finger force-closure grasps for polyhedral objects," IEEE Int. Conf. on Robotics and Automation, pp. 1355-1360, 1995.

[7] J. C. Trinkle, "A quantitative test for form closure grasps," IROS, pp. 1670-1677, 1992.

[8] D. Ding, Y. H. Liu, Y. T. Shen and G. L. Xiang, "An efficient algorithm for computing a 3D form-closure grasp," IROS, 2000.

[9] R. Webster, "Convexity", Oxford Science Publications, 1994.

[10] D. M. Simmons, "Nonlinar programming for operations research", Prentice- Hall International Publications, 1975. 